\title{
Forced Convection Heat Transfer Analysis of Square Shaped Dimples on Flat Plates
}

\author{
Jamil Ahmed ${ }^{1}$, Hasibur Rahman Sardar ${ }^{2}$, Abdul Razak Kaladgi ${ }^{3, *}$ \\ ${ }^{1}$ Department of Computer science and Engineering, P.A College of Engineering, VTU, Mangalore, India \\ ${ }^{2}$ Department of Electronics \& Communication Engineering, P.A College of Engineering, VTU, Mangalore, India \\ ${ }^{3}$ Department of Mechanical Engineering, P.A College of Engineering, VTU, Mangalore, India \\ Email address: \\ jamil.pace@gmail.com (J. Ahmed), hasibpace@gmail.com (H. R. Sardar), abdulkaladgi@gmail.com (A. R. Kaladgi)
}

\section{To cite this article:}

Jamil Ahmed, Hasibur Rahman Sardar, Abdul Razak Kaladgi. Forced Convection Heat Transfer Analysis of Square Shaped Dimples on Flat Plates. American Journal of Energy Engineering. Vol. 3, No. 5, 2015, pp. 66-70. doi: 10.11648/j.ajee.20150305.11

\begin{abstract}
Dimples play a very important role in heat transfer enhancement of electronic cooling systems. This work mainly deals with experimental investigation of forced convection heat transfer over square shaped dimples on a flat aluminum plate under external laminar flow conditions. Experimental measurements on heat transfer rate and friction characteristics of air (with various inlet flow rates) on a flat plate were conducted. Both staggered and inline arrangements of the dimples were considered for the analysis. From the obtained results, it has been observed that the heat transfer coefficient were high for the plate having dimples.
\end{abstract}

Keywords: Forced Convection, Dimples, Friction Coefficient, Passive Techniques

\section{Introduction}

The development of integrated electronic devices with increase level of miniaturization, higher performance and output has increased the cooling requirement of chips considerably. And as the chip temperature increases, the stability and efficiency issues increases so the problem of heat dissipation has become a bottleneck for the development of chips in the electronic industry [1].Passive heat transfer enhancement techniques are used in electronic cooling devices. In these techniques passive augmented devices such as rib-turbulators, concavities (dimples) Extended surfaces or fins, dimples, and protrusions are used. Among these, the dimples (Special concavities) can be considered important because they not only enhances (augument) the heat transfer rate but also produce minimum pressure drop penalties which is important for pumping power requirements[1]. The dimple usually produces vortex pairs, causes flow separation, creates reattachment zones and hence increases the heat transfer rate. And as they do not protrude into the flow so they contribute less to the foam drag, to produce minimum pressure drop penalties [2]. Another advantage is that in dimple manufacture the removal of material takes place and reduces the cost and weight of the equipments.

Kuethe [3] can be considered as the first person to use dimples on flat surfaces. He observed that the dimples promote rapid or turbulent mixing in the flow, acts as vortex generator $\&$ hence enhance the heat transfer. Afanasyev et al [4] carried out an experimental investigation on friction and heat transfer on surfaces having spherical dimples. They used totally ten plates for the investigation and the experiment was carried out for turbulent flow condition. They observed an increase of $30-40 \%$ in the heat transfer rate with no significant effect on the hydrodynamics of flow. In an another study, Chyu et al [5] used the transient liquid crystal imaging system to analyze and compare tear drop type and hemispherical dimples to study the heat transfer distribution in the channel. He observed a considerable increment in the heat transfer rate for the surfaces having dimples (about 2.5 times their smooth counterparts $10,000 \leq \mathrm{Re} \leq 50,000)$. Mahmood et al [6], experimentally investigated the effect of dimples on heat transfer augmentation. They used the flow visualization techniques and concluded that the periodic nature of shedding off of vortices is the main cause of enhancement of heat transfer and is much more pronounced at the downstream rims of the dimples. Mahmood et al [7] studied the effect of Reynolds number, aspect ratio, and temperature ratio \& flow structure in a channel having dimples at one wall. They observed through the flow visualization techniques that the vortices that are shed off from the dimples become stronger as the non-dimensional 
channel height to dimple diameter $(\mathrm{H} / \mathrm{D})$ ratio decreases and increases the local Nusselt number in these regions. Xie et al [8] carried out a numerical investigation to study the effect of different types of heat transfer enhancing devices such as circular fins, protrusions, \& hemispherical dimples mounted on tip wall. They concluded that though the dimples have a simple geometry but they are best suited for cooling of blade tip especially at low Reynolds numbers.

From the literature, it is very much clear that dimples (vortex generators) have high potential to enhance the heat transfer along with the production of lower pressure drop penalties. The other advantages include low weight and cost and low fouling rates [9], However, most of the researchers conducted numerical or experimental work on spherical dimples of uniform diameter [5, 10]. Also most of the research is confined to flow in the channel or Internal flow, with a very few studies on external flow [10].So the main aim of this project is to experimentally study the effect of square dimples under external laminar forced flow conditions. Both staggered and inline arrangements of the dimples are considered for the analysis.

\section{Experimental Setup}

The prime objective of the present work is to study experimentally the heat transfer enhancement through square dimples on aluminum flat plate's using forced convection technique. For this to be possible we required a forced convection setup which was fabricated as required. The fabricated setup is shown below.

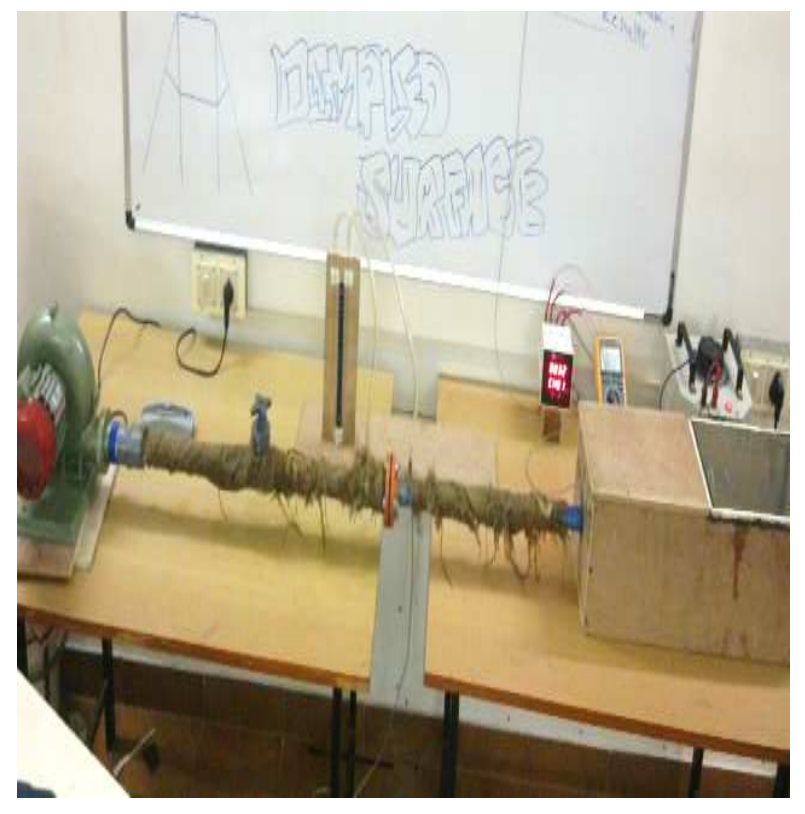

Figure 1. Experimental setup.

The main components of the test apparatus are a test plates of dimensions $100 \times 100 \times 2 \mathrm{~mm}$, a calibrated orifice flow meter, Strip plate heater with capacity of 100 watts, Dimmer stat, Digital temperature, voltmeter, and ammeter with J type thermocouple, a gate valve, and a centrifugal blower. Strip plate heater was used to provide heat input to the test surface. The provision was made to fix the heater at the base of the test plate \& was connected to the blower through an orifice plate of $12 \mathrm{~mm}$ diameter at inlet and to the atmosphere at outlet. U-TUBE manometer was connected across the orifice plate to indicate the pressure difference in terms of centimeters of water column difference. A PVC pipe was used to connect the blower outlet to the rectangular duct. Next to the blower outlet, flow regulating valve was connected to the pipe to regulate the air flow. Orifice plate was introduced next to the regulating valve to measure the regulated air flow rate in the pipe. Air flows parallel to the dimpled test surface. The strip plate heater fixed at the bottom of the test plate, was connected to power socket through dimmer stat. Dimmer stat provides the required heat input to the test plate. A Calibrated J-thermocouple was used to measure the temperatures. Provisions were made to fix the thermocouples on the test surface. Temperatures of air at inlet and outlet of the heat exchange module are also measured. Digital temperature indicators were used to show the temperature readings $\left(\right.$ in ${ }^{\circ} \mathrm{C}$ ) recorded by thermocouple wires. Only top dimpled surface of the test plate was exposed to the air stream from which the convective heat transfer to the air stream takes place. The regulator was used to vary manometer head to give different trails for different shapes of dimples like Square, Triangular and Circular. The experiment was carried out by varying the inlet flow rates, thus varying the Reynolds number with air as a working fluid.

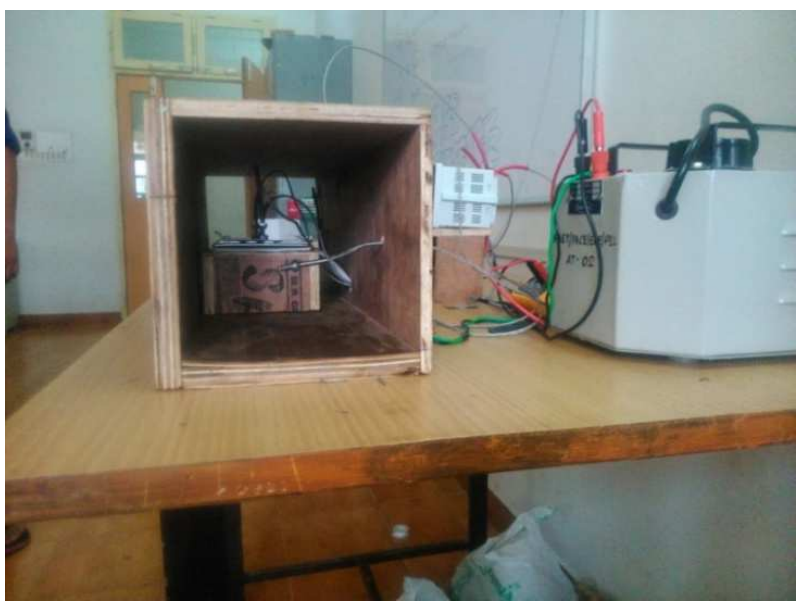

Figure 2. Schematic representation.

Table 1. Components and Specifications.

\begin{tabular}{ll}
\hline Components & Specification \\
\hline Test plate & $10 \times 10 \times 2 \mathrm{~cm}$ Aluminium plates \\
Blower & $110 \mathrm{~W}, 0.4 \mathrm{BHP}, 280 \mathrm{rpm}$ \\
Heater & $100 \mathrm{~W}, 4$ "x4" \\
Dimmer stat & $6 \mathrm{~A}, 230 \mathrm{~V}$ \\
Digital Temperature & 6 channel,12000C, 230V \\
Indicator & $12 \mathrm{~mm}$ dia. \\
Orifice plate & "U-tube" glass manometer \\
Manometer & A wooden casing of size of 8"x8" and 2feet long. \\
Casing & K-Type, 3000C, 1m long. \\
Thermocouple & Voltmeter, Ammeter \\
Digital Multi-meter &
\end{tabular}




\section{Data Reduction}

The study was carried out under Steady laminar external forced convection regime. Steady state values for a given heat flux was used to determine the heat transfer performance parameters.

The various formula used are as follows

- Surface temperature of the plate: $\mathrm{Ts}_{\mathrm{s}}=\left(\mathrm{T}_{2}+\mathrm{T}_{3}+\mathrm{T}_{4}\right) / 3$

- Film temperature $T_{f}=\left(T_{\infty}+T_{s}\right) / 2$

Properties of air at " $\mathrm{T}_{\mathrm{f}}$ " is used to find parameters like

- Prandtl number(Pr)

- Thermal conductivity(k)

- $\operatorname{Density}(\rho)$

- Kinematic viscosity $(v)$

- Reynolds number $=\mathrm{u} * \mathrm{~L} / \mathrm{v}$

Where "u" is air flow velocity, " $\mathrm{L}$ " is the length of aluminum test plate.

- Air flow velocity "u" =Volumetric flow rate/cross sectional area of pipe,

- Volumetric flow rate $=c_{d}\left(\sqrt{ } 2 g H w \rho_{w} / \rho_{a}-1\right) / \sqrt{ }\left(1-m^{2}\right)$

From heat transfer data book, Nusselt number can be related by,

- $\mathrm{Nu}=0.453 \mathrm{Re}^{0.5} \mathrm{Pr}^{0.333}$

- Heat transfer coefficient, $h=\mathrm{Nu} \times \mathrm{k}_{\text {air }} / \mathrm{L}$

Where " $L$ " is the length of test plate.

- Heat transfer rate, $Q=h A_{s}\left(T_{s}-T_{f}\right)$

Where "As" is surface area of aluminum test plate.

\section{Results and Discussion}

Experiments were conducted on Aluminum test plates with square shaped dimples. Inline and staggered arrangements were used for the study. The data obtained were used to find heat transfer parameters like Nusselt number, heat transfer coefficient, heat transfer rate and friction coefficient. And the experimental findings have been plotted in the form of graphs, mainly

- Nusselt number(Nu) vs Reynolds number(Re)

- Heat transfer coefficient(h) vs Reynolds number(Re)

- Heat transfer rate Q vs Reynolds number(Re)

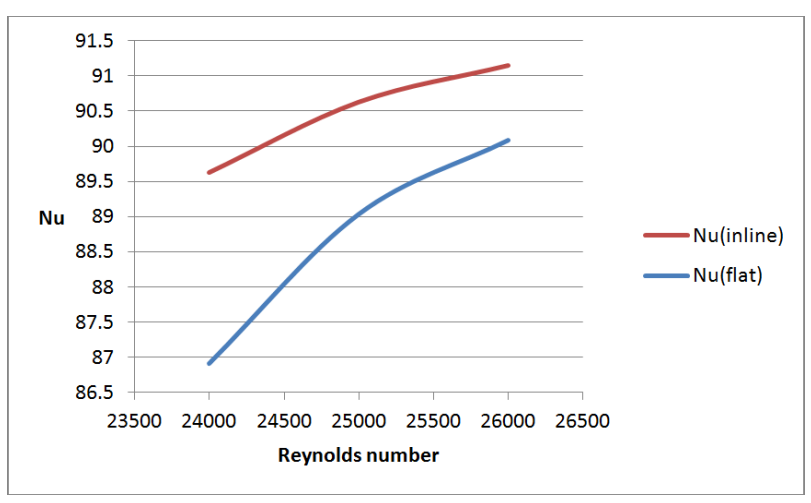

Figure 3. Variation of Nusselt number with Reynolds number(Inline).

Figure $3 \& 4$ shows variation of Nusselt number ' $\mathrm{Nu}$ ' with Reynolds number for the square shaped dimples considered. It is obvious that the ' $\mathrm{Nu}$ ' increases as Reynolds number increases due to direct flow impingement on the downstream boundary and strengthened flow mixing by vortices at the downstream $[1,11]$. The formation of vortex pairs which are periodically shed off from the dimples, a large up wash regions with some fluids coming out from the central regions of the dimples are the main causes of enhancement of Nusselt number $\&$ is more pronounced near the downstream rims of the dimples [6].It can also be seen that the variation in the Nusselt number is gradual with Reynolds number as expected $[12,13,16]$.

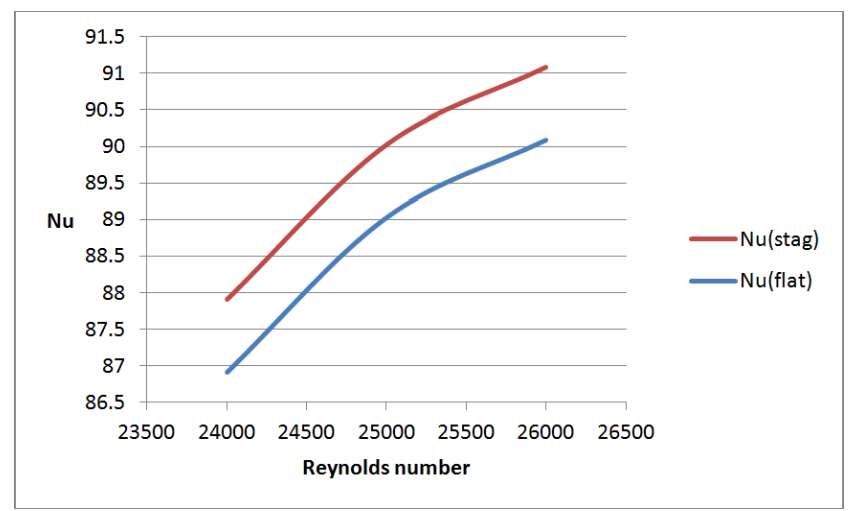

Figure 4. Variation of Nusselt number with Reynolds number(Staggered).
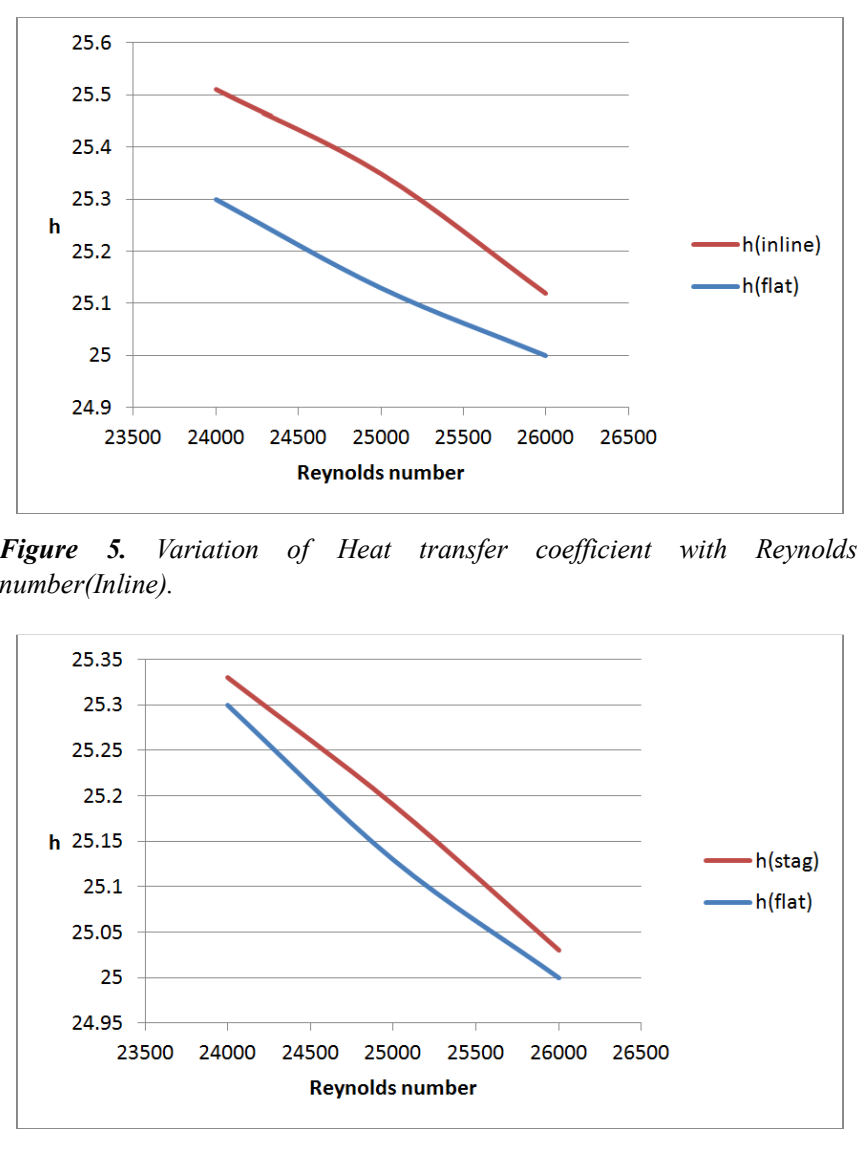

Figure 6. Variation of Heat transfer coefficient with Reynolds number(Staggered).

Figure $5 \& 6$ shows the variation of heat transfer coefficient ' $h$ ' with Reynolds number 'Re' for the square shaped dimple considered. It is obvious that ' $h$ ' increases 
with 'Re'as expected because the development of the thermal boundary layer is delayed or disrupted $\&$ hence enhances the local heat transfer in the reattachment region and wake region and increases the heat transfer coefficient [1].

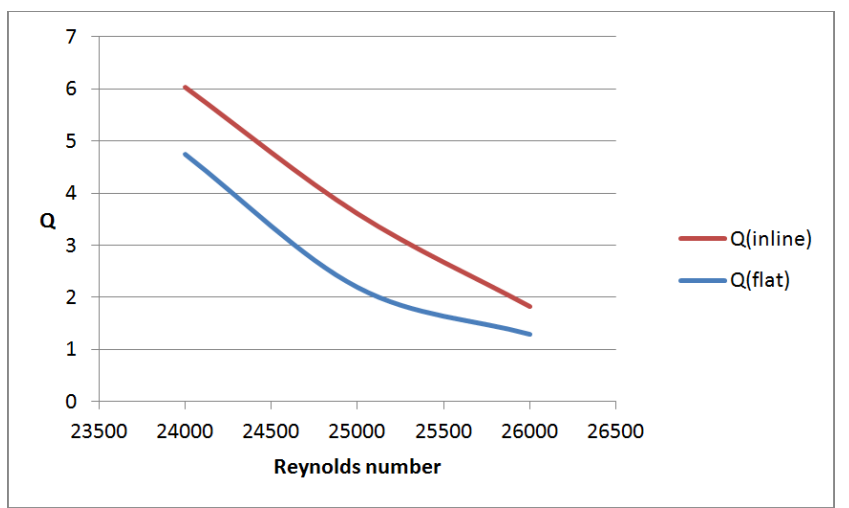

Figure 7. Variation of Heat transfer rate with Reynolds number(Inline).

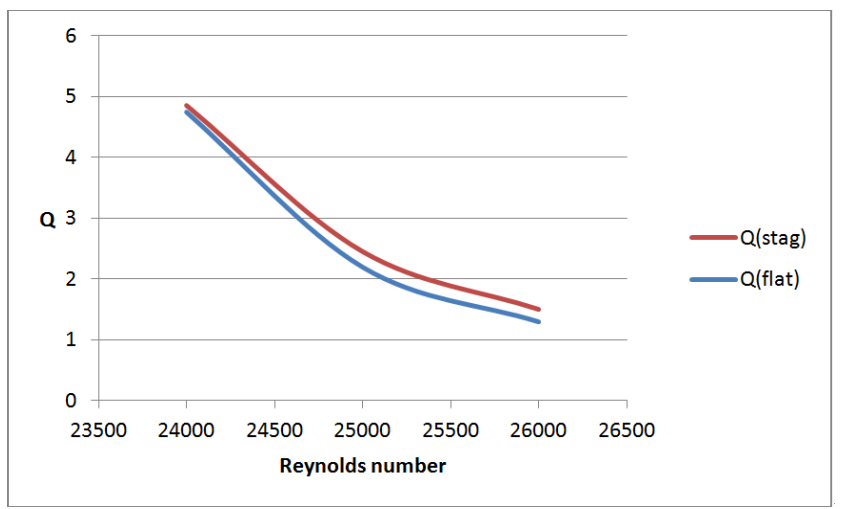

Figure 8. Variation of Heat transfer rate with Reynolds number(Staggered).

Figure $7 \& 8$ shows variation of Heat transfer rate ' $Q$ ' with Reynolds number 'Re' for the square shaped dimples considered. It can be seen that again ' $Q$ ' increases as ' $R e$ ' increases for both the cases i.e. flat plate with dimples and without dimples. It can also be seen that ' $Q$ ' is very much higher for plates having dimples because of increased flow area as compared to flat plates without dimples. Hence it can be concluded that dimples helps in better enhancing the heat transfer compared to flat plates without dimples [8].

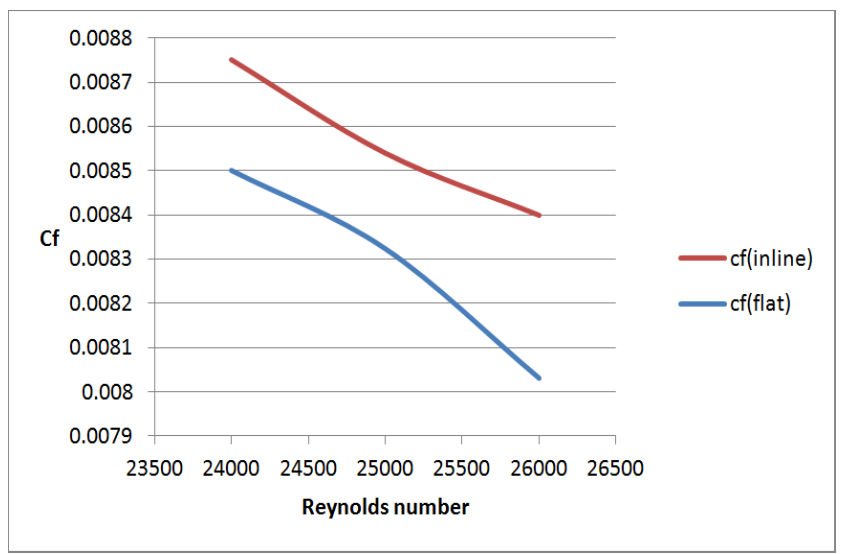

Figure 9. Variation of friction coefficient with Reynolds number(Inline).

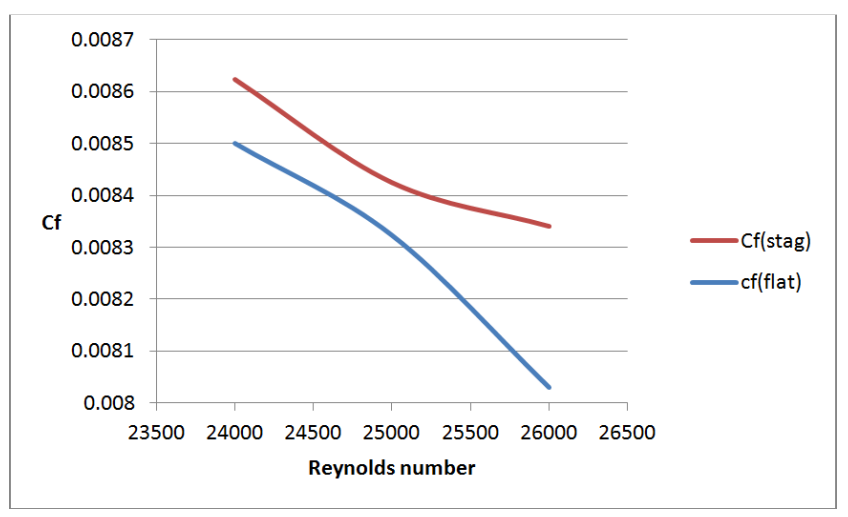

Figure 10. Variation of friction coefficient with Reynolds number(Staggered).

Figure $9 \& 10$ shows variation of average friction coefficient ' $\mathrm{Cf}$ ' with Reynolds number ' $\mathrm{Re}$ ' for the square shaped dimples considered. It can be seen that ' $\mathrm{Cf}$ ' decreases as 'Re' increases for both the cases i.e. flat plate with dimples and without dimples because of the increased flow rates. It can also be seen that ' $\mathrm{Cf}$ ' is little higher for plates having dimples indicating that the enhancement of heat transfer is at the cost of pressure loss. Hence it can be concluded that dimples helps in better enhancing the heat transfer but the pumping requirement may a little high as compared to flat plates.

\section{Conclusion}

In this experimental work an investigation of the effect of air flow over a flat plate with square shaped dimples is carried out. The main conclusions of the work are:

- Nusselt number increases with Reynolds number for the dimple arrangement considered due to direct flow impingement on the downstream boundary and strengthened flow mixing by the vortices at the downstream.

- Heat transfer coefficient increases with Reynolds number for the dimple arrangement considered due to the disruption of the thermal boundary layer development \& hence enhance the local heat transfer in the reattachment and wake regions.

- It can be seen that ' $\mathrm{Q}$ ' increases as ' $\mathrm{Re}$ ' increases for both the cases i.e. flat plate with dimples and without dimples. It can also be seen that ' $Q$ ' is very much higher for plates having dimples because of increased flow area as compared to flat plates without dimples. Hence it can be concluded that dimples helps in better enhancing the heat transfer compared to flat plates without dimples However, the Augmentation depends on the configuration[9].

- It can also be seen that ' $\mathrm{Cf}$ ' is little higher for plates having dimples indicating that the enhancement of heat transfer is at the cost of pressure loss. 


\section{References}

[1] Zhang, D., Zheng, L., Xie, G., and Xie, Y., An Experimental Study on Heat Transfer enhancement of Non-Newtonian Fluid in a Rectangular Channel with Dimples/Protrusions, Transactions of the ASME, Vol. 136, pp.021005-10,2014.

[2] Beves, C.C., Barber, T.J., and Leonardi, E., An Investigation of Flow over Two-Dimensional Circular Cavity. In 15th Australasian Fluid Mechanics Conference, the University of Sydney, Australia, pp.13-17, 2004.

[3] Kuethe A. M., Boundary Layer Control of Flow Separation and Heat Exchange. US Patent No. 1191, 1970.

[4] Afanasyev, V.N., Chudnovsky, Y.P., Leontiev, A.I., and Roganov, P.S., Turbulent flow friction and heat transfer characteristics for spherical cavities on a flat plate. Experimental Thermal Fluid Science, Vol. 7, Issue 1, pp. 1-8, 1993.

[5] Chyu, M.K., Yu, Y., Ding, H., Downs, J.P., and Soechting, F.O., Concavity enhanced heat transfer in an internal cooling passage. In Orlando international Gs Turbine \& Aero engine Congress \& Exhibition, Proceedings of the 1997(ASME paper 97-GT-437), 1997.

[6] Mahmood, G.I., Hill, M.L., Nelson, D.L., Ligrani, P.M., Moon, H.K., and Glezer, B., Local heat transfer and flow structure on and above a dimpled surface in a channel. J Turbo mach, Vol.123, Issue 1, pp: 115-23, 2001.

[7] Mahmood, G. I., and Ligrani, P. M., Heat Transfer in a Dimpled Channel: Combined Influences of Aspect Ratio, Temperature Ratio, Reynolds Number, and Flow Structure. Int. J. Heat Mass Transfer, Vol. 45, pp.2011-2020, 2002.

[8] Xie, G. N., Sunden, B., and Zhang, W. H., Comparisons of Pins/Dimples Protrusions Cooling Concepts for an Internal Blade Tip-Wall at High Reynolds Numbers. ASME J. Heat Transfer, Vol. 133, Issue 6, pp. 0619021-0619029, 2011.

[9] Gadhave, G., and Kumar. P. Enhancement of forced Convection Heat Transfer over Dimple Surface-Review. International Multidisciplinary e - Journal .Vol-1, Issue-2, pp. 51-57, 2012

[10] Katkhaw, N., Vorayos, N., Kiatsiriroat, T., Khunatorn, Y., Bunturat, D., and Nuntaphan. A. Heat transfer behavior of flat plate having $45^{\circ}$ ellipsoidal dimpled surfaces. Case Studies in Thermal Engineering, vol.2, pp. 67-74,2014

[11] Patel, I.H., and Borse, S.H.Experimental investigation of heat transfer enhancement over the dimpled surface. International Journal of Engineering Science and Technology, Vol.4, Issue6, pp.3666-3672, 2012. 